\title{
Suppression of endotoxin-induced inflammation by taxol
}

\author{
T. Mirzapoiazova*, I.A. Kolosova\#, L. Moreno*, S. Sammani*, \\ J.G.N. Garcia* and A.D. Verin"
}

ABSTRACT: The pathogenesis of acute lung injury includes transendothelial diapedesis of leukocytes into lung tissues and disruption of endothelial/epithelial barriers leading to proteinrich oedema. In vitro studies show that the microtubule network plays a role in the regulation of endothelial permeability as well as in neutrophil locomotion. It was hypothesised that the microtubule-stabilising agent, taxol, might attenuate inflammation and vascular leak associated with acute lung injury in vivo.

The effect of intravenously delivered taxol was assessed using a model of murine lung injury induced by intratracheal lipopolysaccharide (LPS) administration. Parameters of lung injury and inflammation were assessed $18 \mathrm{~h}$ after treatment.

Intravenously delivered taxol significantly reduced inflammatory histological changes in lung parenchyma and parameters of LPS-induced inflammation: infiltration of proteins and inflammatory cells into bronchoalveolar lavage fluid, lung myeloperoxidase activity, and extravasation of Evans blue-labelled albumin into lung tissue. Taxol alone (in the absence of LPS) had no appreciable effect on these parameters. In addition to lung proteins, intravenous taxol reduced accumulation of leukocytes in ascitic fluid in a model of LPS-induced peritonitis.

Taken together, the present data demonstrate that microtubule stabilisation with taxol systemically attenuates lipopolysaccharide-induced inflammation and vascular leak.

KEYWORDS: Endotoxin, inflammation, lipopolysaccharide, lung injury, oedema, taxol

A cute lung injury (ALI) and its more severe form, acute respiratory distress syndrome (ARDS), are associated with high morbidity and mortality in patients. The pathogenesis of ALI includes injury of endothelial and epithelial barriers leading to protein-rich oedema, and inflammation induced by cytokines and chemokines released from inflammatory cells, lung epithelial cells or fibroblasts. Weakening of the endothelial barrier enhances the transendothelial diapedesis of leukocytes into lung tissues, further contributing to pulmonary dysfunction.

Endothelial cell barrier regulation is dependent upon an intact cytoskeleton. Although the importance of actin microfilaments for the endothelial barrier is well established [1], involvement of another cytoskeletal component, the tubulin-based microtubule network, has only recently been explored. The capacity of inhibitors of microtubule polymerisation to increase transendothelial permeability to solutes in vitro has been reported [2]. Conversely, microtubule stabilisation by taxol attenuates solute permeability across the endothelial monolayer and paracellular gap formation induced by tumour necrosis factor (TNF)- $\alpha$ [3] and thrombin [4]. Furthermore, stabilisation of endothelial microtubules with taxol decreases leukocyte transmigration through endothelial monolayers, whereas disassembly of microtubules increases leukocyte migration [5]. Microtubules may also be involved directly in neutrophil locomotion, since pre-treatment with taxol markedly inhibits neutrophil chemotaxis stimulated by endotoxin-activated serum [6].

These in vitro data suggest that microtubule stabilisation may reduce microvascular permeability to solutes and leukocytes in vivo. Therefore, it was hypothesised that microtubule stabilisation might decrease vascular leakage and inflammation in the acutely injured lung. In order to test this hypothesis, the effect of taxol in a murine model of endotoxin-induced lung injury was examined. Endotoxin/lipopolysaccharide (LPS), a key constituent of Gram-negative bacterial cell walls, is a pro-inflammatory mediator and, when delivered intratracheally, reproducibly displays key features of microvascular lung injury, including leukocyte accumulation in lung tissue, pulmonary oedema, profound lung inflammation and mortality $[7,8]$. LPS treatment leads to

\section{AFFILIATIONS}

*Dept of Medicine, The University of Chicago, Chicago, IL,

\#Dept of Medicine, Case Western Reserve University, Cleveland, $\mathrm{OH}$, and

- Vascular Biology Center, Medical College of Georgia, Augusta, GA, USA.

CORRESPONDENCE

A.D. Verin

Vascular Biology Center

CB-3210A

Medical College of Georgia

Augusta

GA $30912-2500$

USA

Fax: 17067219799

E-mail: averin@mcg.edu

Received:

November 272006

Accepted after revision:

May 092007

\section{SUPPORT STATEMENT}

This study was supported by grants from the National Heart, Lung, and Blood Institute (Bethesda, MD, USA: HL80675, HL067307, HL083327 and HL58064).

STATEMENT OF INTEREST

None declared 
disruption of peripheral microtubules in pneumocytes [9] and vascular endothelial cells [10]. In the present study, it is shown that microtubule stabilisation has a beneficial effect on ALI outcome, since intravenous taxol caused marked attenuation of vascular leak and inflammation in a murine model of endotoxin-induced ALI.

\section{MATERIALS AND METHODS}

\section{Animals}

All experiments and animal care procedures were approved by the University of Chicago Animal Resource Center (Chicago, IL, USA) and animals were handled according to the National Institutes of Health Guide for the Care and Use of Laboratory Animals [11]. C57BL/6 (20-25 g) mice were purchased from Jackson Laboratory (Bar Harbor, ME, USA) and housed in cages with access to food and water in a temperaturecontrolled room with a 12 -h dark/light cycle until the time of experiments.

\section{Animal procedures}

Mice were anaesthetised using $150 \mathrm{mg} \cdot \mathrm{kg}^{-1}$ ketamine and $15 \mathrm{mg} \cdot \mathrm{kg}^{-1}$ acetylpromazine before exposure of the trachea and right internal jugular vein via neck incision. LPS solution (2.5 $\mathrm{mg} \cdot \mathrm{kg}^{-1}$ body weight Escherichia coli LPS, serotype 055:B5; Sigma, St Louis, MO, USA) or sterile saline was instilled intratracheally via a 20-gauge catheter. Simultaneously, through the internal jugular vein, mice received a single injection of either taxol (Sigma), to reach a final calculated plasma concentration of $10 \mu \mathrm{M}$, or cremophor/ethanol/PBS control. The animals were allowed to recover for $18 \mathrm{~h}$. Bronchoalveolar lavage fluid (BALF) and lungs were collected and stored at $-70^{\circ} \mathrm{C}$ for further evaluation of lung injury.

\section{Quantification of total protein and leukocyte analysis in BALF}

BALF was centrifuged $\left(15 \mathrm{~min}\right.$ at $1,500 \times g$ at $\left.4^{\circ} \mathrm{C}\right)$ and the supernatant used for measurement of total protein using the BCA Protein Assay Kit (Pierce Chemical Co., Rockford, IL, USA). Cell pellets were examined for the total number of white blood cells using a haemocytometer. In addition, cytospin slides were prepared from cell suspensions. After hemacolor staining (EMD Chemicals, Gibbstown, NJ, USA), differential cell counts of neutrophils and macrophages were performed by counting 300-500 cells using a microscope.
TNF- $\alpha$, interleukin- 6 and prostaglandin $E_{2}$ assays

ELISAs were adapted to measure mouse TNF- $\alpha$ and interleukin (IL)-6 (R\&D Systems, Chantilly, VA, USA) and prostaglandin (PG) $\mathrm{E}_{2}$ (Cayman Chemicals, Ann Arbor, MI, USA) in BALF and performed according to the manufacturers' protocols.

\section{Histopathology}

Lungs were perfused free of blood with PBS and infused with $0.5 \%$ low-melting-point agarose for histological evaluation by haematoxylin and eosin staining.

\section{Measurement of Evans blue-labelled albumin accumulation in lungs}

Evans blue-labelled albumin (EBA) was injected into the jugular vein 120 min before termination of the experiment in order to assess vascular leak, as described previously [8]. After the experiment, lungs were perfused with PBS through the right ventricle, weighed and snap-frozen in liquid nitrogen. EBA was extracted in formamide for $18 \mathrm{~h}$ at $60^{\circ} \mathrm{C}$ from right lungs. The optical density of the supernatants was determined spectrophotometrically at $620 \mathrm{~nm}$. The extravasated EBA concentration in lung homogenate was calculated against a standard curve.

\section{LPS-induced peritonitis}

Acute peritonitis was induced by intraperitoneal injection of LPS $\left(0.2 \mathrm{mg} \cdot \mathrm{kg}^{-1}\right.$ body weight in $100 \mu \mathrm{L}$ PBS). Peritoneal washes were performed $4 \mathrm{~h}$ later using $1 \mathrm{~mL}$ PBS. The collected ascitic fluid was analysed for cellular content using Diff-Quik stain (Dade Behring, Inc., Deerfield, IL, USA).

\section{Statistical analysis}

Data are presented as mean \pm SEM. Data were analysed using standard one-way ANOVA, groups were compared by Newman-Keuls test and significance, in all cases, was defined at $\mathrm{p}<0.05$.

\section{RESULTS \\ Effect of taxol on parameters of LPS-induced lung inflammation}

Histological examination of lung tissue $18 \mathrm{~h}$ after LPS administration indicated inflammatory changes, such as alveolar wall thickening and leukocyte infiltration into the lung interstitium and alveolar space, compared with the saline
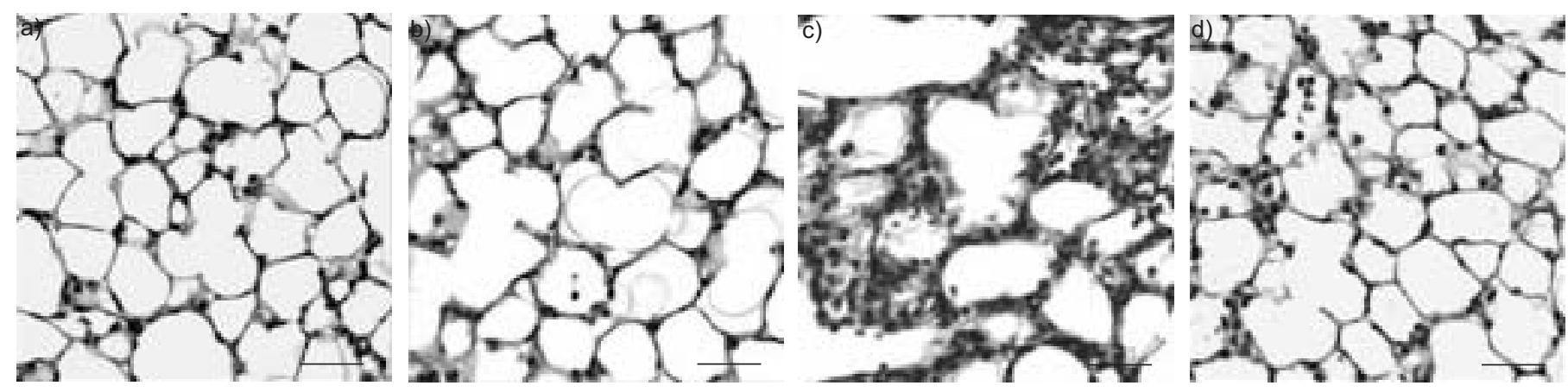

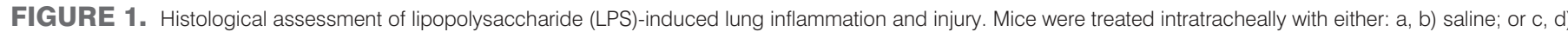

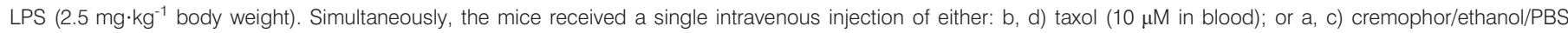
(vehicle). Lungs were harvested $18 \mathrm{~h}$ after the treatments. Scale bar $=50 \mu \mathrm{m}$. 

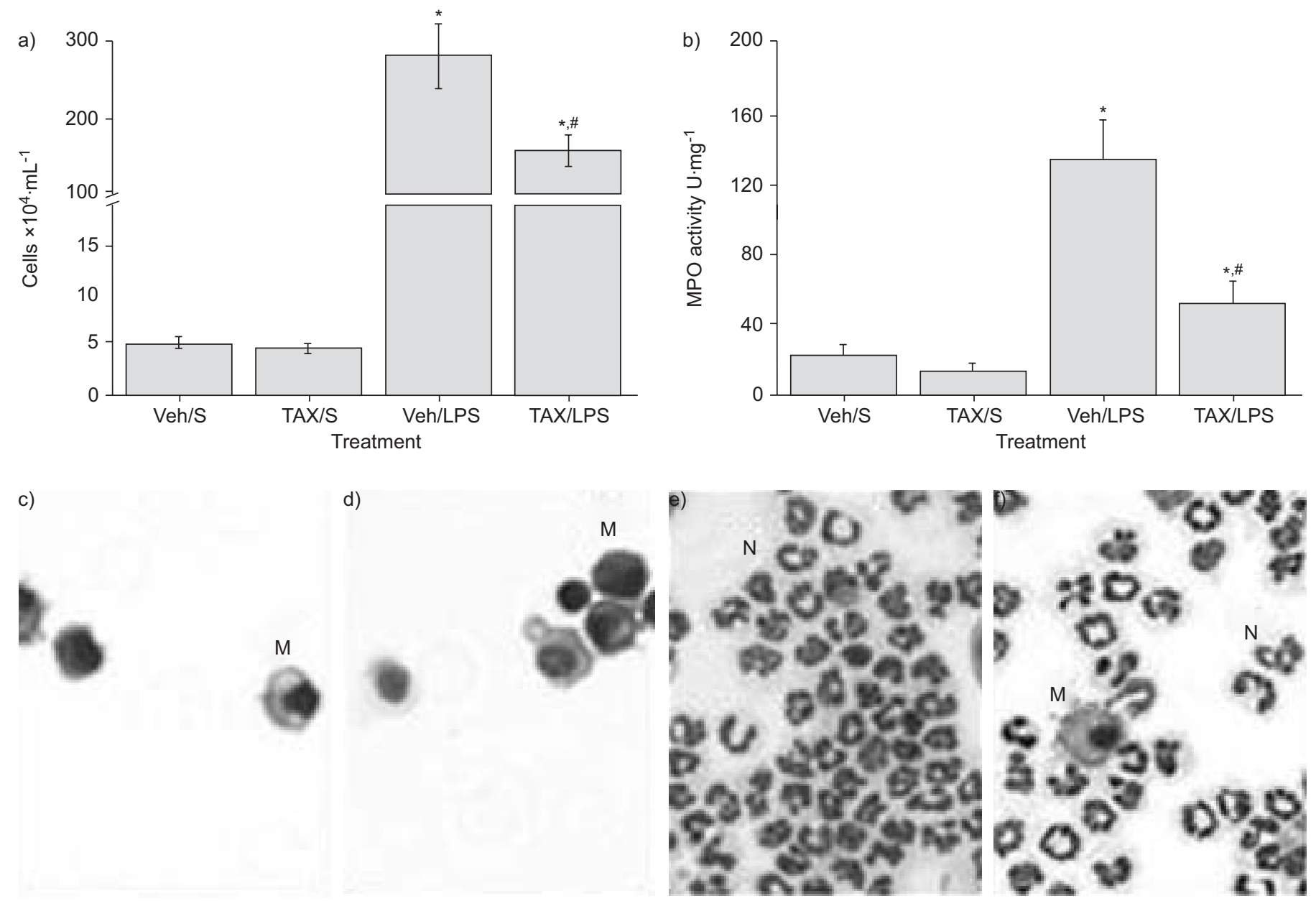

FIGURE 2. Effect of taxol (TAX) on lipopolysaccharide (LPS)-induced accumulation of white blood cells in bronchoalveolar lavage fluid (BALF). a) Total number of cells in BALF. LPS-mediated cell infiltration into lung tissue was reduced by TAX treatment $(n=4-9)$. Vehicle (Veh)/saline (S): $n=4 ;$ TAX/S: $n=6$; Veh/LPS: $n=9 ;$ TAX/LPS: $n=6$. b) Myeloperoxidase (MPO) activity in lung homogenates. MPO activity was elevated in lung tissue of the LPS/Veh group; TAX significantly decreased LPS-induced MPO activity (LPS/TAX group). Veh/S: $n=3$; TAX/S: $n=3$; Veh/LPS: $n=5$; TAX/LPS: $n=4$. Data are presented as mean \pm SEM. *: $p<0.05$ versus $S /$ Veh group; ${ }^{*}: p<0.05$ versus LPS/Veh group. Mice were treated intratracheally with either: $\mathrm{c}, \mathrm{d}) \mathrm{S}$; or e, f) LPS (2.5 $\mathrm{mg} \cdot \mathrm{kg}^{-1}$ body weight). Simultaneously, the mice received a single intravenous injection of either: d, f) TAX (10 $\mu \mathrm{M}$ in blood); or c, e) cremophor/ethanol/PBS (Veh). BALF was collected $18 \mathrm{~h}$ after the treatments. c-f) Hemacolor staining of BALF after cytospin separation. Representative images of BALF from each group are shown. Macrophages (M), but not neutrophils (N), are present in the BALF of the S/Veh and S/TAX groups. LPS induced accumulation of neutrophils in BALF (LPS/Neh group). This accumulation was remarkably reduced by intravenous TAX (LPS/TAX group).

control (fig. 1). Taxol treatment alone did not cause any noticeable changes in lung histology (fig. 1), but greatly diminished histological signs of LPS-induced inflammation (fig. 1). Quantitatively, this effect was confirmed by counting total cells in BALF (fig. 2a). Taxol/LPS-treated mice showed a reduced total number of cells in BALF, compared with those treated with LPS alone. Microscopic examination showed that BALF from saline- and taxol-treated mice mostly contained macrophages, whereas LPS treatment led to increased infiltration of neutrophils (fig. 2b). Lung myeloperoxidase activity, an index of neutrophil sequestration in the lungs, was reduced in taxol-treated mice, consistent with the histological evaluation (fig. 2c). LPS challenge also increased the number of circulating neutrophils in peripheral blood (fig. 3). Prolonged taxol treatment is known to cause neutropenia in mice [12]. However, in the present experiments, the reduction in neutrophil infiltration into the lungs after taxol treatment was not due to neutropenia, since the total number of neutrophils in the blood was not affected by taxol (fig. 3).

\section{Effect of taxol on LPS-induced lung vascular leak}

Pulmonary microvascular leakage induced by LPS was reflected in increased BALF protein concentration compared with saline-challenged mice and mice challenged with taxol alone (fig. 4a). Taxol significantly reduced the BALF protein concentration $(\sim 40 \%$; $<0.001)$ in LPS-treated mice (fig. $4 a)$. LPS exposure induced pulmonary oedema (fig. 4b), as measured by extravasation of EBA into lung parenchyma (fig. 4c). Taxol alone did not alter basal levels of EBA extravasation, but significantly attenuated LPS-induced microvascular lung leakage (figs $4 \mathrm{~b}$ and $\mathrm{c}$ ).

\section{Effect of taxol on cytokine and $P \mathrm{PE}_{2}$ production in lungs}

Many of the systemic effects of LPS are due to the production of cytokines and chemokines in the body. Intratracheal LPS 


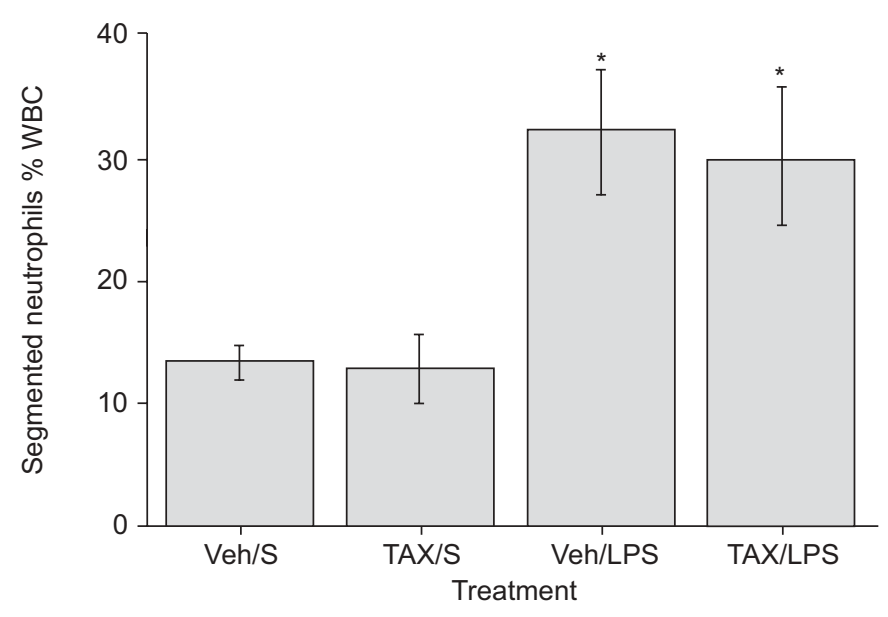

FIGURE 3. Neutrophil content of mouse blood relative to total number of white blood cells (WBC). Mice were treated intratracheally with either lipopolysaccharide (LPS; $2.5 \mathrm{mg} \cdot \mathrm{kg}^{-1}$ body weight) or saline (S). Simultaneously, the mice received a single intravenous injection of either taxol (TAX; $10 \mu \mathrm{M}$ in blood) or cremophor/ ethanol/PBS (vehicle (Veh)). Blood was collected $18 \mathrm{~h}$ after the treatments. Veh/S: $n=3 ;$ TAX/S: $n=3 ;$ Veh/LPS: $n=6$; TAX/LPS: $n=5$. Data are presented as mean \pm SEM. *: $p<0.05$ versus $S / N$ eh group.

administration dramatically increased levels of TNF- $\alpha(2,003 \pm$ $\left.166 \mathrm{pg} \cdot \mathrm{mL}^{-1}\right)$ and IL-6 $\left(451.5 \pm 29.8 \mathrm{pg} \cdot \mathrm{mL}^{-1}\right)$ in BALF, compared with levels in animals treated with saline $\left(2.8 \pm 1.1 \mathrm{pg} \cdot \mathrm{mL}^{-1}\right.$ and $3.9 \pm 2.5 \mathrm{pg} \cdot \mathrm{mL}^{-1}$ for TNF- $\alpha$ and IL-6, respectively; figs $5 \mathrm{a}$ and $\mathrm{b}$ ). Simultaneous LPS/taxol treatment modestly but significantly decreased both TNF- $\alpha$ and IL-6 levels in BALF compared with LPS alone (figs $5 \mathrm{a}$ and $\mathrm{b}$ ).

$\mathrm{PGE}_{2}$ is a pro-inflammatory mediator, although its exact mechanistic role in ALI remains to be investigated. LPS treatment caused increased accumulation of $\mathrm{PGE}_{2}$ in BALF, which was significantly reduced by taxol (fig. 5c). $\mathrm{PGE}_{2}$ levels in BALF from animals treated with taxol alone were lower than control values (fig. 5c).

\section{Effect of taxol on LPS-induced peritonitis}

Sepsis-induced ALI/ARDS can be initiated by acute peritonitis developing in response to Gram-negative bacterial infection. In order to evaluate whether a potentially beneficial effect of intravenous taxol occurs in LPS-induced peritonitis, intraperitoneal LPS was delivered to induce acute peritonitis, reflected in increased recruitment of leukocytes into the peritoneal cavity (fig. 6a). Microscopic examination of ascitic fluid revealed predominance of macrophages (58\%), although neutrophils $(11 \%)$ were also present with a significant amount of erythrocytes (fig. 6b), indicating considerable vascular leak. Intravenous taxol reduced the effects of LPS to basal levels (figs 6a and b). Taxol alone did not alter cell counts or composition in ascitic fluid compared with the saline control (figs $6 a$ and b).

\section{DISCUSSION}

ALI and ARDS are major causes of acute respiratory failure that are associated with high morbidity and mortality. These disorders are characterised by a significant pulmonary inflammatory response, resulting in injury to alveolar epithelial and endothelial barriers of the lung and protein-rich pulmonary oedema. ALI/ARDS pathogenesis is still only partly understood, and its pharmacological treatment is nonspecific. Based on published in vitro data, it was hypothesised that microtubule dynamics may play an important role in ALI pathogenesis and recovery.

The present study in mice with LPS-induced ALI indicated that the microtubule-stabilising agent taxol exerts significant anti-inflammatory and anti-oedemagenic effects. Although the mechanism of this effect needs to be explored, the present authors speculate that the effects of taxol may improve endothelial/epithelial barriers, since taxol significantly attenuates LPS-induced accumulation of water and proteins in BALF and the interstitium. The present data are in agreement with the recent observation that taxol prevents loss of the endothelial barrier in isolated lung [13].

In microvascular endothelium, cell-cell junctions are key to maintaining the integrity of the vessel wall. LPS challenge of cultured endothelial cells results in the loss of monolayer integrity and paracellular gap formation [14, 15]. Microtubule stabilisation by taxol may interfere with LPS effects on cell-cell junctions, since recent data suggest that microtubules and adherence junctions interact dynamically $[16,17]$. Conversely, in LPS-challenged cells, endothelial junctions may be weakened due to contractile forces generated by cytoskeletal motor proteins $[14,18]$. Microtubule stabilisation with taxol may affect the contractile cytoskeleton in a way that preserves cell-cell junctions. Although, the relationship between microtubules and the contractile cytoskeleton is not fully explored, the disruption of either microfilaments or microtubule structure can cause endothelial cell contraction and barrier dysfunction via alterations in the actomyosin cytoskeleton. Microtubule depolymerisation, using either nocodazole or vinblastine, in pulmonary endothelial cells is associated with myosin light-chain phosphorylation, stress fibre formation, contraction and barrier dysfunction $[2,4]$. These effects are linked to activation of the small Rho guanosine triphosphatase and its effector Rho kinase, and can be attenuated by cell pre-treatment with taxol, which promotes microtubule assembly $[2,4]$.

Besides enhancing the endothelial barrier, taxol may affect other components of ALI pathogenesis. Recruitment and activation of neutrophils within the lung capillaries is largely responsible for sepsis-related pulmonary injury [19]. Since taxol inhibits polymorphonuclear neutrophil locomotion in vitro [6], intravenous taxol may reduce the ability of neutrophils to migrate toward the sites of inflammation. The present data show that the number of neutrophils in the lungs and ascitic fluid of LPS-challenged mice is significantly decreased.

TNF- $\alpha$ and other cytokines derived from LPS-stimulated cells mediate the toxic effect of LPS [20]. It was shown that taxol slightly reduced cytokine accumulation in the lungs. However, their level in the BALF of LPS-treated animals was still much higher than that of the control. Therefore, it appears unlikely that cytokine reduction is the major mechanism of taxol action.

Published in vitro data demonstrate that taxol can induce inflammatory signalling in isolated murine macrophages, increasing their TNF- $\alpha$ and IL-1 release [21, 22]. Induction of inflammatory signalling, however, requires a higher taxol concentration $(\sim 30 \mu \mathrm{M})$ than that sufficient for microtubule 

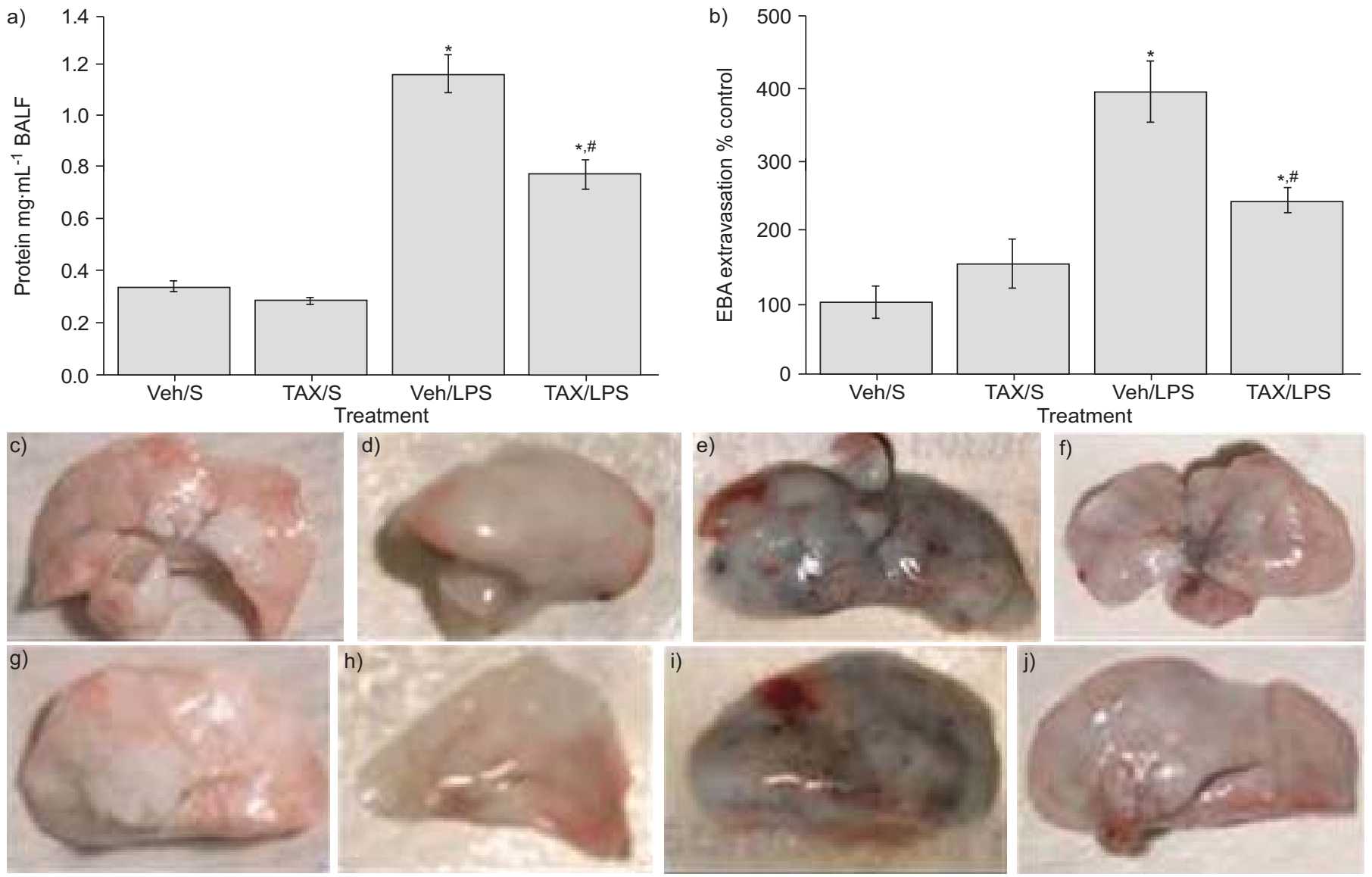

FIGURE 4. Effect of taxol (TAX) on lipopolysaccharide (LPS)-induced pulmonary oedema. a) Total protein accumulation in bronchoalveolar lavage fluid (BALF). The increase in BALF protein produced by LPS challenge was significantly reduced by TAX. Vehicle (Veh)/saline (S): $n=4 ;$ TAX/S: $n=6 ;$ Veh/LPS: $n=9 ;$ TAX/LPS: $n=9$. b) Quantitative analysis of Evans blue-labelled albumin (EBA) extravasation. The EBA content of lung extracts was determined spectrophotometrically. Veh/S: $n=3 ; T A X / S$ : $n=3$; Veh/LPS: $n=7$; TAX/LPS: $n=6$. Data are presented as mean \pm SEM. *: $p<0.05$ versus $S /$ veh group; ${ }^{*}: p<0.05$ versus LPS/Neh group. Mice were treated intratracheally with either: $\mathrm{c}, \mathrm{d}, \mathrm{g}, \mathrm{h}$ ) S; or e, f, i, j) LPS (2.5 mg. $\mathrm{kg}^{-1}$ body weight). Simultaneously, the mice received a single intravenous injection of either: $\left.\mathrm{d}, \mathrm{f}, \mathrm{h}, \mathrm{j}\right)$ TAX (10 $\mu \mathrm{M}$ in blood); or c, e, $\mathrm{g}$, i) cremophor/ethanol/PBS (Veh). Parameters of oedema were assessed $18 \mathrm{~h}$ after the treatments. c-j) EBA extravasation in: c-f) right; and g-i) left lungs. EBA was injected into the jugular vein $120 \mathrm{~min}$ before the termination of the experiment. LPS induced visible EBA leakage from the vascular space into surrounding lung tissue (LPS/Veh group). This leakage was noticeably reduced in TAX-treated mice (LPS/TAX group).
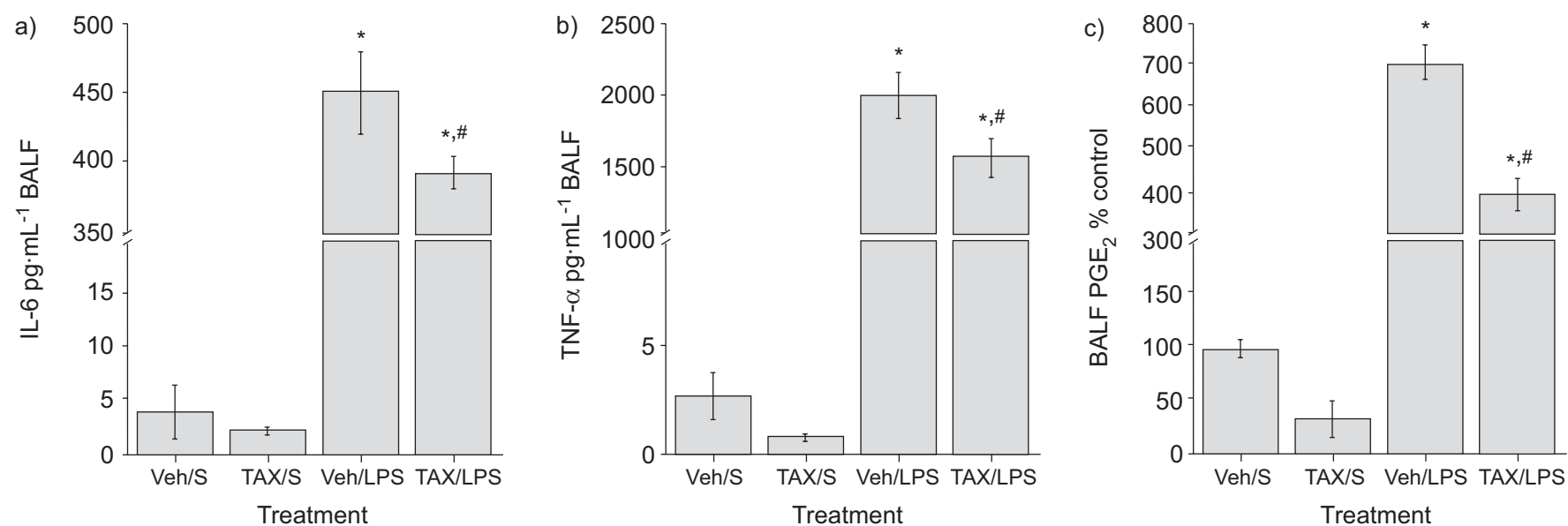

FIGURE 5. Cytokine and prostaglandin (PG) $\mathrm{E}_{2}$ production in lungs. Mice were treated intratracheally with either: saline (S) or LPS (2.5 mg $\cdot \mathrm{kg}^{-1} \mathrm{body}$ weight). Simultaneously, the mice received a single intravenous injection of either taxol (TAX; $10 \mu \mathrm{M}$ in blood) or cremophor/ethanol/PBS (vehicle (Veh)). Bronchoalveolar lavage fluid (BALF) cytokine and prostaglandin levels were measured $18 \mathrm{~h}$ after treatment with: a) interleukin (IL)-6; b) tumour necrosis factor (TNF)- $\alpha$; and c) PGE 2 . Production of all three was attenuated by TAX with or without LPS. Veh/S: $n=3$; TAX/S: $n=4$; Veh/LPS: $n=7 ;$ TAX/LPS: $n=6$. Data are presented as mean \pm SEM. ${ }^{*}$ : $p<0.05$ versus $S /$ Veh group; \#: $p<0.05$ versus LPS/Neh group. 

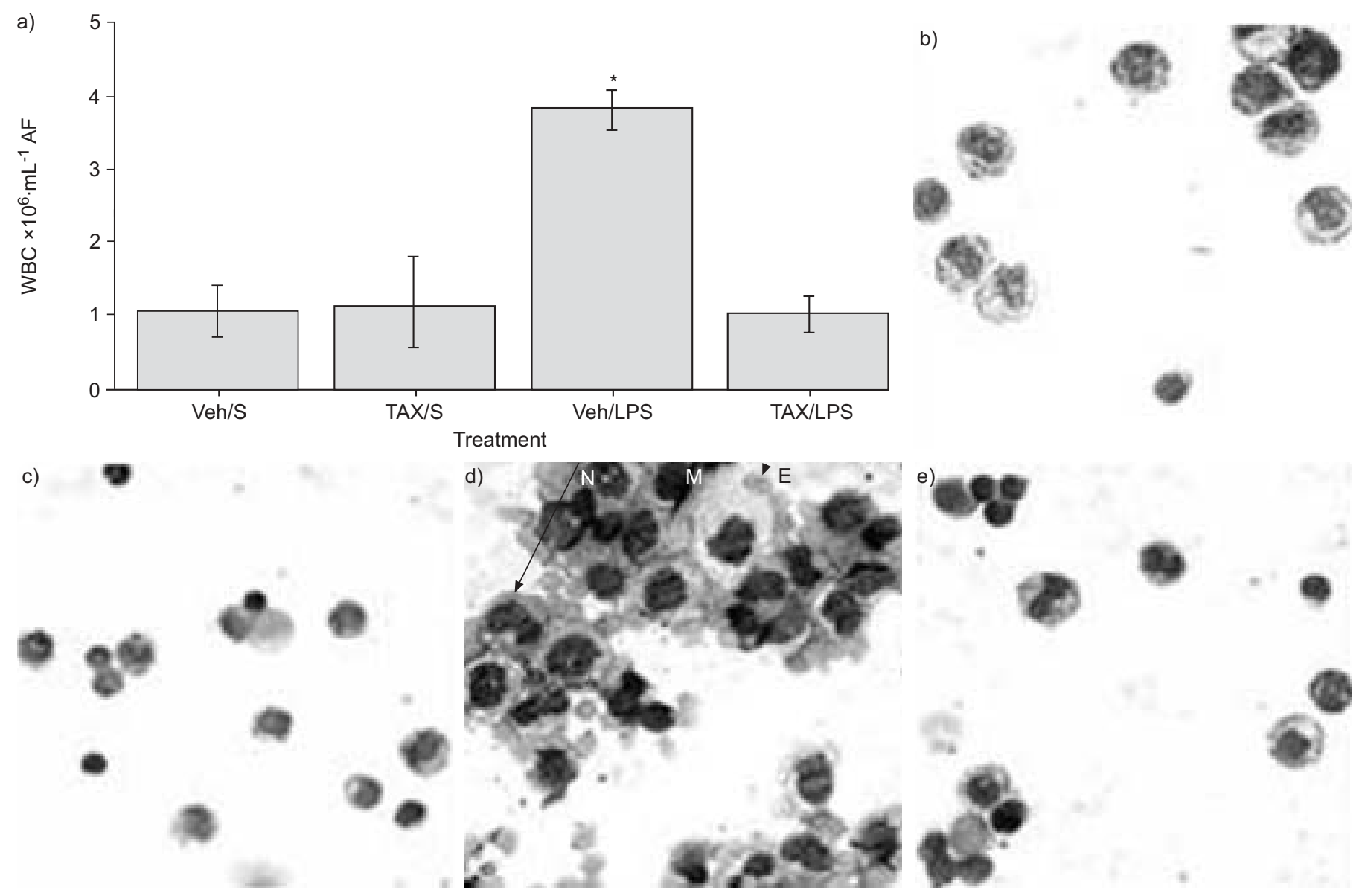

FIGURE 6. Effect of taxol (TAX) on lipopolysaccharide (LPS)-induced acute peritonitis. a) Total number of white blood cells (WBC) in ascitic fluid (AF). LPS significantly increased the number of accumulated WBC in AF (LPS/vehicle (Veh) group) compared to the saline (S)/Neh control. TAX reduced the number of WBC to numbers comparable to the normal level. Veh/S: $n=4 ;$ TAX/S: $n=4$; Veh/LPS: $n=9$; TAX/LPS: $n=9$. Data are presented as mean \pm SEM. *: $p<0.05$ versus $S /$ Veh group. b) Cellular composition of AF after cytospin separation and Diff-Quik staining. Representative images of AF from each group are shown. Mice received an intraperitoneal injection of either: b, c) S; or d, e) LPS ( $0.2 \mathrm{mg} \cdot \mathrm{kg}^{-1}$ body weight). Simultaneously, the mice received a single intravenous injection of either: c, e) TAX (10 $\mu \mathrm{M}$ in blood); or b, d) cremophor/ethanol/PBS (Veh). AF was collected $4 \mathrm{~h}$ after the treatments. Intraperitoneal LPS caused accumulation of polymorphonuclear neutrophils (N), macrophages (M), and erythrocytes (E) in AF. The AF composition of the LPS/TAX group was comparable to that of the S/Neh group. TAX alone did not change AF composition compared to the S/Neh group.

stabilisation $(10 \mu \mathrm{M})$, which was used in the present study. Furthermore, taxol may still inhibit cytokine production and secretion by other pulmonary cell types, including epithelial and endothelial cells, resulting in a net decrease in pulmonary cytokine accumulation. Importantly, studies on breast cancer patients indicate that taxanes (paclictaxel and docetaxel) decrease levels of the serum cytokines IL- 1 and TNF- $\alpha$ and increase levels of interferon- $\gamma$, IL-2 and IL-6 [23].

$\mathrm{PGE}_{2}$ is a potent immunosuppressive eicosanoid, levels of which are significantly elevated in the plasma of septic patients [24] and animals [25], with the majority of plasma $\mathrm{PGE}_{2}$ produced in the lungs [26]. Although the role of $\mathrm{PGE}_{2}$ in ALI remains to be investigated, lung inflammation in mice and rats is accompanied by increased levels of $\mathrm{PGE}_{2}$ in $\operatorname{BALF}[27,28]$. In the present system, taxol significantly attenuated $\mathrm{PGE}_{2}$ accumulation in the BALF of LPS-treated mice. This is in agreement with the observation that taxanes reduce plasma levels of $\mathrm{PGE}_{2}$ in humans [23].
The involvement of microtubules in key signal transduction pathways has been accepted [29], with the polymerisation dynamics of microtubules being central to their biological functions [30]. Tubulin-binding drugs, such as colchicines and taxol, strongly modulate microtubule dynamics. Although colchicine inhibits microtubule polymerisation, taxol stimulates it and stabilises microtubules, completely eliminating the soluble pool of tubulin within the cell. As a result, both of these drugs cause cell cycle arrest. It has previously been established that the microtubule-depolymerising agent, colchicine, has a significant anti-inflammatory effect. Its mode of action includes modulation of chemokine and prostanoid production and inhibition of neutrophil and endothelial cell adhesion molecules [31]. The present data indicate, for the first time, that microtubule stabilisation with taxol results in an anti-inflammatory effect, indicating that not only the structural integrity of microtubules but also microtubule dynamics are important in inflammatory signalling. 


\section{REFERENCES}

1 Dudek SM, Garcia JG. Cytoskeletal regulation of pulmonary vascular permeability. J Appl Physiol 2000; 91: 1487-1500.

2 Verin AD, Birukova A, Wang P, et al. Microtubule disassembly increases endothelial cell barrier dysfunction: role of MLC phosphorylation. Am J Physiol Lung Cell Mol Physiol 2001; 281: L565-L574.

3 Petrache I, Birukova A, Ramirez SI, Garcia JG, Verin AD. The role of the microtubules in tumor necrosis factor- $\alpha$ induced endothelial cell permeability. Am J Respir Cell Mol Biol 2003; 28: 574-581.

4 Birukova AA, Smurova K, Birukov KG, et al. Microtubule disassembly induces cytoskeletal remodeling and lung vascular barrier dysfunction: role of Rho-dependent mechanisms. J Cell Physiol 2004; 201: 55-70.

5 Kielbassa K, Schmitz C, Gerke V. Disruption of endothelial microfilaments selectively reduces the transendothelial migration of monocytes. Exp Cell Res 1998; 243: 129-141.

6 Roberts RL, Nath J, Friedman MM, Gallin JI. Effects of taxol on human neutrophils. J Immunol 1982; 129: 2134-2141.

7 Demling RH. The modern version of adult respiratory distress syndrome. Annu Rev Med 1995; 46: 193-202.

8 Peng X, Hassoun PM, Sammani S, et al. Protective effects of sphingosine 1-phosphate in murine endotoxin-induced inflammatory lung injury. Am J Respir Crit Care Med 2004; 169: 1245-1251.

9 Isowa N, Xavier AM, Dziak E, et al. LPS-induced depolymerization of cytoskeleton and its role in TNF- $\alpha$ production by rat pneumocytes. Am J Physiol Lung Cell Mol Physiol 1999; 277: L606-L615.

10 Chakravortty D, Koide N, Kato Y, et al. Cytoskeletal alterations in lipopolysaccharide-induced bovine vascular endothelial cell injury and its prevention by sodium arsenite. Clin Diagn Lab Immunol 2000; 7: 218-225.

11 Institute of Laboratory Animal Research, Commission on Life Sciences, National Research Council. Guide for the Care and Use of Laboratory Animals. Washington, DC, National Academies Press, 1996.

12 Juaristi JA, Aguirre MV, Carmuega RJ, Romero-Benitez M, Alvarez MA, Brandan NC. Hematotoxicity induced by paclitaxel: in vitro and in vivo assays during normal murine hematopoietic recovery. Methods Find Exp Clin Pharmacol 2001; 23: 161-167.

13 Suzuki S, Bing H, Sugawara T, et al. Paclitaxel prevents loss of pulmonary endothelial barrier integrity during cold preservation. Transplantation 2004; 78: 524-529.

14 Goldblum SE, Ding X, Brann TW, Campbell-Washington J. Bacterial lipopolysaccharide induces actin reorganization, intercellular gap formation, and endothelial barrier dysfunction in pulmonary vascular endothelial cells: concurrent F-actin depolymerization and new actin synthesis. J Cell Physiol 1993; 157: 13-23.

15 De Vries HE, Blom-Roosemalen MC, de Boer AG, van Berkel TJ, Breimer DD, Kuiper J. Effect of endotoxin on permeability of bovine cerebral endothelial cell layers in vitro. J Pharmacol Exp Ther 1996; 277: 1418-1423.

16 Waterman-Storer CM, Salmon WC, Salmon ED. Feedback interactions between cell-cell adherens junctions and cytoskeletal dynamics in newt lung epithelial cells. Mol Biol Cell 2000; 11: 2471-2483.

17 Chausovsky A, Bershadsky AD, Borisy GG. Cadherinmediated regulation of microtubule dynamics. Nat Cell Biol 2000; 2: 797-804.

18 Essler M, Staddon JM, Weber PC, Aepfelbacher M. Cyclic AMP blocks bacterial lipopolysaccharide-induced myosin light chain phosphorylation in endothelial cells through inhibition of Rho/Rho kinase signaling. J Immunol 2000; 164: 6543-6549.

19 Lee WL, Downey GP. Neutrophil activation and acute lung injury. Curr Opin Crit Care 2001; 7: 1-7.

20 Beutler B, Kruys V. Lipopolysaccharide signal transduction, regulation of tumor necrosis factor biosynthesis, and signaling by tumor necrosis factor itself. J Cardiovasc Pharmacol 1995; 25: Suppl. 2, S1-S8.

21 Ding AH, Porteu F, Sanchez E, Nathan SF. Shared actions of endotoxin and taxol on TNF receptors and TNF release. Science 1990; 248: 370-372.

22 Bogdan C, Ding A. Taxol, a microtubule-stabilizing antineoplastic agent, induces expression of tumor necrosis factor $\alpha$ and interleukin-1 in macrophages. J Leukoc Biol 1992; 52 : 119-121.

23 Tsavaris N, Kosmas C, Vadiaka M, Kanelopoulos P, Boulamatsis D. Immune changes in patients with advanced breast cancer undergoing chemotherapy with taxanes. $\mathrm{Br} \mathrm{J}$ Cancer 2002; 87: 21-27.

24 Faist E, Mewes A, Baker CC, et al. Prostaglandin $\mathrm{E}_{2}\left(\mathrm{PGE}_{2}\right)$ dependent suppression of interleukin-2 production in patients with major trauma. J Trauma 1987; 27: 837-848.

25 Yao YM, Wang YP, Tian HM, et al. Reduction of circulating prostaglandin $\mathrm{E}_{2}$ level by antiserum against core lipopolysaccharide in a rabbit model of multiple organ failure. J Trauma 1996; 40: 270-277.

26 Piper PJ, Vane JR, Wyllie JH. Inactivation of prostaglandins by the lungs. Nature 1970; 225: 600-604.

27 Steil AA, Tavares de Lima W, Jancar S. Modulation by lipid mediators of immune complex-induced lung inflammation in mice. Eur J Pharmacol 1998; 361: 93-99.

28 Alba-Loureiro TC, Martins EF, Miyasaka CK, et al. Evidence that arachidonic acid derived from neutrophils and prostaglandin $\mathrm{E}_{2}$ are associated with the induction of acute lung inflammation by lipopolysaccharide of Escherichia coli. Inflamm Res 2004; 53: 658-663.

29 Gundersen GG, Cook TA. Microtubules and signal transduction. Curr Opin Cell Biol 1999; 11: 81-94.

30 Desai A, Mitchison TJ. Microtubule polymerization dynamics. Annu Rev Cell Dev Biol 1997; 13: 83-117.

31 Molad Y. Update on colchicine and its mechanism of action. Curr Rheumatol Rep 2002; 4: 252-256. 